Volume 2

Issue 4 -- Integrative Medicine

Article 19

$11-20-2015$

\title{
A Meta-Analysis of Incidence and Risk Factors of Trastuzumab- Induced Cardiotoxicity in Breast Cancer
}

\author{
Zeeshan Ali Jawa \\ Ruth M. Perez \\ Lydia Garlie \\ Maharaj Singh \\ Rubina Qamar \\ Bijoy K. Khandheria \\ Arshad Jahangir \\ Yang Shi
}

Follow this and additional works at: https://aah.org/jpcrr

Part of the Cardiology Commons, Chemicals and Drugs Commons, and the Oncology Commons

\section{Recommended Citation}

Jawa Z, Perez RM, Garlie L, Singh M, Qamar R, Khandheria BK, Jahangir A, Shi Y. A Meta-Analysis of Incidence and Risk Factors of Trastuzumab-Induced Cardiotoxicity in Breast Cancer. J Patient Cent Res Rev 2015;2:207-208. http://dx.doi.org/10.17294/2330-0698.1229

Published quarterly by Midwest-based health system Advocate Aurora Health and indexed in PubMed Central, the Journal of Patient-Centered Research and Reviews (JPCRR) is an open access, peer-reviewed medical journal focused on disseminating scholarly works devoted to improving patient-centered care practices, health outcomes, and the patient experience. 
allocating palliative care services.

Purpose: To systematically review and summarize current medical literature regarding the factors predictive of mortality in an inpatient population above 65 years of age.

Methods: Nondisease-specific prognostication indices that predict 1-year mortality in an inpatient population of adults over age 65 were included. We excluded studies that estimated intensive care unit, disease-specific or in-hospital mortality. A MEDLINE, CINAHL, Ovid and Cochrane literature search of English-language articles that developed and/or validated a prognostication index to predict mortality was performed. Review of 3,600 citations revealed 53 articles that reported variables associated with mortality. Based on the inclusion criteria, 9 studies were included in the final analysis. Data was extracted from the 9 studies using the following parameters: adequate method of description of population, nonbiased selection of patients, low loss to follow-up, adequate prognostic factor measurements, adequate outcome measurements and methods of validation. We performed qualitative analysis on 5 studies and 4 studies were pooled for a quantitative meta-analysis.

Results: The 1-year mortality rate for the 21,338 patients included in all the studies was 31\% (95\% confidence interval [CI]: 31.3-32.6); mean age was 80.6 years. Factors significantly associated with mortality included male sex (odds ratio [OR]: $1.25,95 \%$ CI: 1.09-1.42; $\mathrm{P}<0.001$ ), congestive heart failure (OR: $0.41,95 \% \mathrm{CI}$ : 0.37-0.45; $\mathrm{P}<0.001$ ), chronic obstructive pulmonary disease (OR: 3.2, 95\% CI: $0.42-24.9 ; \mathrm{P}=0.26)$, myocardial infarction (proportion $0.39 ; \mathrm{P}<0.001$ ), and cerebrovascular disease (proportion 0.38, 95\% CI: 0.32-0.44; $\mathrm{P}<0.001$ ).

Conclusion: One-year mortality for inpatients aged > 65 years was high and associated with male sex, chronic obstructive pulmonary disease and congestive heart failure. Generalization of these findings to all older adults should be made with caution because of insufficient published information. In the future, our results may be used to develop a prognostication tool that draws patient data in real time from the EHR to identify vulnerable older adults in the hospital with end-of-life needs.

\section{Chronic Illness Management in Teams of Urban Multidisciplinary Scholars (CIMTUMS) - Part II}

John R. Brill, Diane Ames, Christine B. Groth, Helen Yu

Academic Affairs, Aurora UW Medical Group; Concordia University; Pharmacy, Aurora Health Care; University of Wisconsin School of Medicine and Public Health

Background: Diabetes is a major contributor to morbidity and mortality as well as the single most expensive health care condition in the world. Numerous interventions have attempted to improve control of this disorder and reduce its complications. Traditional care for diabetes centers on an individual clinician. More recently, recognition of the central role of the patient has come into vogue. Payors, including Medicare, now cover up to 13 hours of diabetes self-management and education programs annually. Patientcentered medical home efforts add an aspect of inclusiveness, but retain a medical focus and are being increasingly advocated and trained. To date, no research has focused on the use of interprofessional learning teams simultaneously delivering care and learning to work together.

Purpose: This project contributes to the development and training of interprofessional learner teams to enhance patient care. Intended outcomes include learner attitude and behavior changes and improvement in diabetic patients' biomarkers, empowerment and satisfaction.

Methods: Teams of 6-9 learners from eight fields and three universities work with cohorts of 6-21 African-American diabetic patients. The project includes team training, implementation of a diabetes self-management education (DSME) program and weekly telephone coaching. Learners complete the Centers for Disease Control and Prevention's TeamSTEPPS teamwork attitude questionnaire pre- and postintervention. Patients are recruited from the Aurora Midtown Clinic, which serves a largely Medicare/Medicaid population in Milwaukee's central city. Registries are searched for patients who fall out of quality goals; they are recruited by team members, with a target of 25 to 30 willing patients to complete the five DSME sessions and 4-week phone coaching around SMART goals. Patient biomarker data is tabulated, and pre- and postintervention Diabetes Empowerment Scale completed.

Results: Three cohorts of 42 patients and 23 students have completed the program. Patients demonstrated high attendance rates, improved diabetes knowledge and selfmanagement skills, and a trend in improvement in diabetes control compared to age/gender-matched controls. Students did not show a change in interprofessional attitude.

Conclusion: Challenges included coordinating schedules, demonstrating change of attitude in self-selected students, and discipline-specific supervision requirements. These programs demonstrated feasibility of concept for an interprofessional student-led DSME program to enhance patient care, with high student interest and engagement.

\section{A Meta-Analysis of Incidence and Risk Factors of Trastuzumab-Induced Cardiotoxicity in Breast Cancer}

Zeeshan Ali Jawa, Ruth M. Perez, Lydia Garlie, Maharaj Singh, Rubina Qamar, Bijoy K. Khandheria, Arshad Jahangir, Yang Shi

Internal Medicine, Medical College of Wisconsin; Aurora Research Institute, Aurora Health Care; University of Wisconsin-Madison; Medical Oncology, Aurora Health Care; Aurora Cardiovascular Services, Aurora Health Care; Sheikh Khalifa bin Hamad Al Thani Center for Integrative Research on Cardiovascular Aging, Aurora Health Care

Background: A monoclonal antibody, trastuzumab targets the human epidermal growth factor receptor 2 (HER2) 
oncogene that is overexpressed in $25-30 \%$ of breast cancers. In combination with first-line therapy, trastuzumab resulted in significant improvement in survival outcomes for those with HER2-positive metastatic breast cancer. Due to its improvement in outcome and prolonged survival, trastuzumab has been established as standard of care in both adjuvant and metastatic settings. However, along with common adverse events, trastuzumab has been found to be associated with cardiotoxicity. An estimated $1-4 \%$ of patients treated with trastuzumab will develop heart failure and $\sim 10 \%$ of patients will experience a reduction in left ventricular ejection fraction (LVEF). Many studies have published on the risk factors of trastuzumab-induced cardiotoxicity (TIC), with some discrepancy. Whereas one study found that of all risk factors accounted for (age, hypertension, LVEF, radiotherapy) only age was significantly associated with TIC, another found that LVEF was the sole factor, and others found that a combination of these were indicative of TIC.

Purpose: This paper aims to consolidate the data and identify potential risk factors from combined data.

Methods: A computer-based literature search using MEDLINE database was executed using the keywords trastuzumab/Herceptin, risk factors, outcomes, cardiac, cardiotoxicity, cardiomyopathy, LVEF and chemotherapy. Only prospective/retrospective human studies were included, with additional studies excluded if they reported a baseline LVEF $>68$, a cohort $<50$ patients, and/or results were not stratified based on cadiotoxic events.

Results: Data was collected from 17 articles, capturing 6,527 patients. A familial history of cardiac disease (odds ratio [OR]: $3.31,95 \%$ confidence interval [CI]: 1.80-6.08; $\mathrm{P}<0.01$ ), diagnoses of hypertension (OR: $1.61,95 \% \mathrm{CI}$ : 1.14-2.26; $\mathrm{P}<0.01$ ), diabetes (OR: 1.62, 95\% CI: 1.1-2.38; $\mathrm{P}=0.014$ ), and previous anthracycline use (OR: $2.14,95 \%$ CI: $1.17-3.92 ; \mathrm{P}=0.013)$ were all shown to be associated with TIC. Age $(\mathrm{P}=0.013)$ also was a risk factor.

Conclusion: Additional measures need to be set in place for monitoring cardiac performance in women treated with trastuzumab. Being aware of the potential risk factors along with careful attention to symptoms/LVEF can hopefully minimize the occurrence of TIC in this population.

\section{Delirium Recognition in Hospitalized Older Patients: A Quality Improvement Project}

\section{Jodi Punke, Ariba Khan, Michael L. Malone}

\section{Department of Geriatrics, Aurora Sinai Medical Center and Aurora UW Medical Group}

Background: We noted a low reported prevalence of delirium $(3 \%)$ in hospitalized older patients at a community teaching hospital in north central Wisconsin.

Purpose: This was a quality improvement project to report recognition of delirium by nurses before and after an educational intervention.

Methods: This project was performed on one medical unit in our hospital. Quality improvement data was collected at baseline and after the educational intervention. Data collected included observation by a geriatrician attending weekly interdisciplinary rounds to note any mention by nurses of delirium or confusion. The patient's electronic health record (EHR) was reviewed to note delirium assessment by "confusion assessment method for the intensive care unit (Vanderbilt)" (CAM-ICU) by the nurses for 2 days prior to the team meeting. The numbers of positive and total attempted CAMICU were recorded. Use of antipsychotics or benzodiazepines was reported as a "delirium marker." Diagnosis of delirium and dementia was obtained from the problem list in the EHR. The educational intervention included Just-in-Time Teaching during weekly Acute Care for Elders rounds during a 1-month period.

Results: In month 1, before intervention, CAM-ICU was performed 140 times in 2 days on 32 patients with an average CAM-ICU performed 2.2 times per patient/day. There were 3 concerning quotes for confusion during team rounds and 0 for delirium by nurses during team rounds. EHR review noted 7 patients had dementia, 2 had a positive CAM-ICU and 3 had a diagnosis of delirium. In month 2, after intervention, CAM-ICU was performed 163 times in 2 days on 35 patients with an average CAM-ICU performed 2.35 times per patient/ day. There were 6 concerning quotes regarding confusion and 1 regarding delirium by nurses during team rounds. EHR review noted 1 patient had dementia, 0 had a positive CAM-ICU and 0 patients with delirium diagnosis.

Conclusion: This quality improvement project using Justin-Time Teaching by a geriatrician during weekly rounds resulted in a modest increase in number of times CAMICU was performed, increased discussion of delirium during rounds, but no increase in delirium recognition using CAM-ICU. Areas for improvement include involving more physicians and nursing staff along with more structured delirium education.

\section{Stent Thrombosis: Regional Prevalence, Risk Factors, and Outcomes}

Andrew M. Ayers, Chi C. Cho, Robyn Shearer, M. Fuad Jan, Anjan Gupta

Aurora Cardiovascular Services, Aurora Health Care; Aurora Research Institute, Aurora Health Care

Background: Stent thrombosis is an infrequent but catastrophic complication of percutaneous coronary intervention (PCI). Many studies usually involve few stent thrombosis patients, generally less than 60 , given its prevalence. While dual antiplatelet therapy has decreased stent thrombosis significantly in the general population, there are still patients who present with occurrence and recurrence of stent thrombosis.

Purpose: We sought to define the prevalence of site-specific stent thrombosis in a larger cohort of patients by specific coronary territories and determine if this had an effect on cardiovascular outcomes. In addition we sought to elucidate the role of previous coronary artery bypass grafting (CABG) 